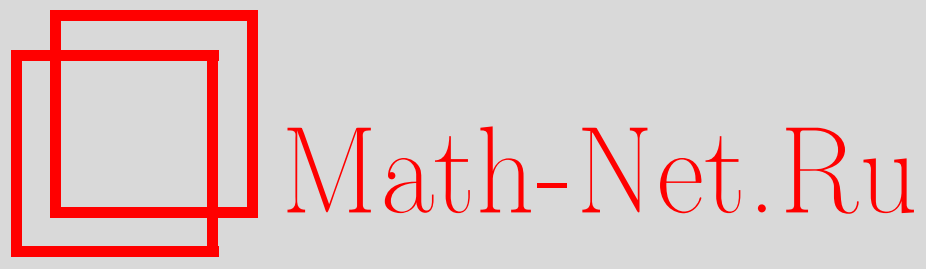

Л. Г. Рыбников, Метод сдвига инвариантов и модель Годена, Функи. анализ и его прил., 2006, том 40, выпуск $3,30-43$

DOI: https://doi.org/10.4213/faa741

Использование Общероссийского математического портала Math-Net.Ru подразумевает, что вы прочитали и согласны с пользовательским соглашением

http://www . mathnet.ru/rus/agreement

Параметры загрузки:

IP: 54.166 .219 .16

26 апреля 2023 г., 11:33:25

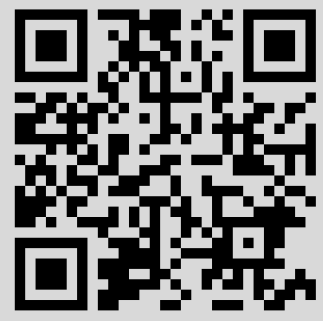


Функииональньй анализ и его приложения

2006, т. 40, вып. 3, с. 30-43

УДК 512.813.4

\title{
Метод сдвига инвариантов и модель Годена*
}

\author{
(c) 2006. Л. Г. Рыьников
}

\section{$\S 1$. Введение}

Пусть $\mathfrak{g}$ - полупростая комплексная алгебра Ли и $U(\mathfrak{g})$ - ее универсальная обертывающая алгебра. На алгебре $U(\mathfrak{g})$ имеется фильтрация по степени выражения через образующие. По теореме Пуанкаре-Биркгофа-Витта ассоциированная градуированная алгебра есть симметрическая алгебра $S(\mathfrak{g})=\mathbb{C}\left[\mathfrak{g}^{*}\right]$. Коммутатор в $U(\mathfrak{g})$ задает скобку Пуассона-Ли в $S(\mathfrak{g})$.

Метод сдвига инвариантов позволяет строить коммутативные относительно скобки Пуассона-Ли подалгебры в $S(\mathfrak{g})$. Этот метод состоит в следующем. Пусть $Z S(\mathfrak{g})=S(\mathfrak{g})^{\mathfrak{g}}$ - центр алгебры $S(\mathfrak{g})$ относительно скобки Пуассона, и пусть $\mu \in \mathfrak{g}^{*}-$ регулярный полупростой элемент. Тогда подалгебра $A_{\mu} \subset S(\mathfrak{g})$, порожденная элементами вида $\partial_{\mu}^{n} \Phi$, где $\Phi \in Z S(\mathfrak{g})$ (или, что то же самое, центральными элементами алгебры $S(\mathfrak{g})=\mathbb{C}\left[\mathfrak{g}^{*}\right]$, сдвинутыми на $t \mu$ при всевозможных $t \in \mathbb{C}$ ), коммутативна относительно скобки Пуассона и имеет максимально возможную степень трансцендентности, равную $\frac{1}{2}(\operatorname{dim} \mathfrak{g}+\mathrm{rk} \mathfrak{g})$ (см. [10]). Более того, подалгебры $A_{\mu}$ являются максимальными коммутативными подалгебрами в $S(\mathfrak{g})$ относительно скобки Пуассона-Ли [17]. Подалгебры $A_{\mu} \subset S(\mathfrak{g})$ названы в [19] подалгебрами Мищенко-Фоменко.

В данной работе мы поднимем подалгебры $A_{\mu} \subset S(\mathfrak{g})$ до коммутативных подалгебр в универсальной обертывающей алгебре $U(\mathfrak{g})$. Иначе говоря, для любой полупростой алгебры Ли $\mathfrak{g}$ мы построим семейство коммутативных подалгебр $\mathscr{A}_{\mu} \subset U(\mathfrak{g})$, параметризованное регулярными полупростыми элементами $\mu \in \mathfrak{g}^{*}$, таких, что gr $\mathscr{A}_{\mu}=A_{\mu}$. Для классических алгебр Ли $\mathfrak{g}$ это было сделано (другими методами) Ольшанским и Назаровым (см. [14], [11]), а в случае $\mathfrak{g}=s l_{r}$ также Тарасовым [16].

Наша конструкция является модификацией известной конструкции высших гамильтонианов модели Годена (см. [7], [3]). Модель Годена была введена в [8] как спиновая модель, связанная с алгеброй Ли $s l_{2}$, и обобщена на случай произвольной полупростой алгебры Ли в [9, п. 13.2.2]. Эта обобщенная модель Годена допускает следующую алгебраическую интерпретацию. Пусть $V_{\lambda}$ - неприводимое представление алгебры Ли $\mathfrak{g}$ со старшим весом $\lambda$. Для любого набора $(\lambda)=$ $\left(\lambda_{1}, \ldots, \lambda_{n}\right)$ целочисленных доминантных весов положим $V_{(\lambda)}=V_{\lambda_{1}} \otimes \cdots \otimes V_{\lambda_{n}}$. Для любого $x \in \mathfrak{g}$ рассмотрим оператор $x^{(i)}=1 \otimes \cdots \otimes 1 \otimes x \otimes 1 \otimes \cdots \otimes 1$ ( $x$ стоит на $i$-м месте), действующий в пространстве $V_{(\lambda)}$. Пусть $\left\{x_{a}\right\}, a=1, \ldots, \operatorname{dim} \mathfrak{g},-$ opтонормированный относительно формы Киллинга базис в $\mathfrak{g}$, и пусть $z_{1}, \ldots, z_{n}-$ набор попарно различных комплексных чисел. Гамильтонианами модели Годе-

*Работа выполнена при поддержке фондов CRDF, грант RM1-2543-MO-03, и РФФИ, грант 05-01-00988-а. 
на являются следующие коммутирующие операторы, действующие в пространстве $V_{(\lambda)}$ :

$$
H_{i}=\sum_{k \neq i} \sum_{a=1}^{\operatorname{dim} \mathfrak{g}} \frac{x_{a}^{(i)} x_{a}^{(k)}}{z_{i}-z_{k}} .
$$

Гамильтонианы $H_{i}$ можно рассматривать как элементы алгебры $U(\mathfrak{g})^{\otimes n}$. В [7] был предложен способ построения большой коммутативной подалгебры $\mathscr{A}\left(z_{1}, \ldots, z_{n}\right) \subset U(\mathfrak{g})^{\otimes n}$, содержащей $H_{i}$. В случае $\mathfrak{g}=s l_{2}$ алгебра $\mathscr{A}\left(z_{1}, \ldots, z_{n}\right)$ порождается элементами $H_{i}$ и центральными элементами алгебры $U(\mathfrak{g})^{\otimes n}$, в остальных случаях в алгебре $\mathscr{A}\left(z_{1}, \ldots, z_{n}\right)$ появляются еще и новые образующие, которые принято называть высшими гамильтонианами Годена. Конструкция алгебры $\mathscr{A}\left(z_{1}, \ldots, z_{n}\right)$ использует следующий глубокий факт [4]: пополненная универсальная обертывающая алгебра аффинной алгебры Каца-Муди $\hat{\mathfrak{g}}$ на критическом уровне имеет большой центр $Z(\hat{\mathfrak{g}})$. Каждому набору комплексных чисел $z_{1}, \ldots, z_{n}$ естественным образом сопоставляется гомоморфизм $Z(\hat{\mathfrak{g}}) \rightarrow U(\mathfrak{g})^{\otimes n}$. Алгебра $\mathscr{A}\left(z_{1}, \ldots, z_{n}\right)$ есть образ этого гомоморфизма.

Мы построим семейство гомоморфизмов $Z(\hat{\mathfrak{g}}) \rightarrow U(\mathfrak{g})^{\otimes n} \otimes S(\mathfrak{g})$, параметризованное наборами попарно различных комплексных чисел $z_{1}, \ldots, z_{n}$. Для каждого набора $z_{1}, \ldots, z_{n}$ образом такого гомоморфизма является некоторая коммутативная подалгебра $\mathscr{A}\left(z_{1}, \ldots, z_{n}, \infty\right) \subset U(\mathfrak{g})^{\otimes n} \otimes S(\mathfrak{g})$. Специализация в точке $\mu \in \mathfrak{g}^{*}=\operatorname{Spec} S(\mathfrak{g})$ дает коммутативную подалгебру $\mathscr{A}_{\mu}\left(z_{1}, \ldots, z_{n}\right) \subset U(\mathfrak{g})^{\otimes n}$, зависящую от $z_{1}, \ldots, z_{n}$ и $\mu \in \mathfrak{g}^{*}$. При $n=1$ получаются коммутативные подалгебры $\mathscr{A}_{\mu}\left(z_{1}\right)=\mathscr{A}_{\mu} \subset U(\mathfrak{g})$, не зависящие от $z_{1}$. Мы докажем, что gr $\mathscr{A}_{\mu}=A_{\mu}$ для регулярных полупростых $\mu$, т. е. подалгебры $\mathscr{A}_{\mu} \subset U(\mathfrak{g})$ являются поднятиями подалгебр Мищенко-Фоменко. При $\mu=0$ имеем $\mathscr{A}_{0}\left(z_{1}, \ldots, z_{n}\right)=\mathscr{A}\left(z_{1}, \ldots, z_{n}\right)$, т. е. подалгебры $\mathscr{A}_{0}\left(z_{1}, \ldots, z_{n}\right) \subset U(\mathfrak{g})^{\otimes n}$ порождены (высшими) гамильтонианами Годена. Мы докажем, что при общих значениях параметров $z_{1}, \ldots, z_{n}$ и $\mu$ подалгебры $\mathscr{A}_{\mu}\left(z_{1}, \ldots, z_{n}\right)$ имеют максимально возможную степень трансцендентности. Эти подалгебры содержат следующие «неоднородные гамильтонианы Годена»:

$$
H_{i}=\sum_{k \neq i} \sum_{a=1}^{\operatorname{dim} \mathfrak{g}} \frac{x_{a}^{(i)} x_{a}^{(k)}}{z_{i}-z_{k}}+\sum_{a=1}^{\operatorname{dim} \mathfrak{g}} \mu\left(x_{a}\right) x_{a}^{(i)} .
$$

Основным вопросом, связанным с моделью Годена, является вопрос о совместной диагонализации (высших) гамильтонианов Годена. Этому вопросу посвящено огромное количество литературы (см. например $[5,6,7,12])$. Из конструкции, приведенной в [7], следует, что подалгебра $\mathscr{A}\left(z_{1}, \ldots, z_{n}\right) \subset U(\mathfrak{g})^{\otimes n}$ состоит из элементов, инвариантных относительно диагонального действия алгебры Ли $\mathfrak{g}$, и поэтому достаточно диагонализировать алгебру $\mathscr{A}\left(z_{1}, \ldots, z_{n}\right)$ в подпространстве $V_{(\lambda)}^{\text {sing }} \subset V_{(\lambda)}$ особых векторов относительно $\operatorname{diag}_{n}(\mathfrak{g})$ (т. е. относительно диагонального действия алгебры Ли g). Стандартная гипотеза состоит в том, что спектр алгебры $\mathscr{A}\left(z_{1}, \ldots, z_{n}\right)$ в пространстве $V_{(\lambda)}^{\text {sing }}$ прост при общих значениях $z_{i}$. Эта гипотеза доказана в [12] для $\mathfrak{g}=s l_{r}$ при условии, что $\lambda_{i}$ равны $\omega_{1}$ или $\omega_{r-1}$ (т. е. когда каждое $V_{\lambda_{i}}$ есть стандартное представление алгебры Ли $s l_{r}$ или двойственное ему).

Естественно также поставить вопрос о диагонализации алгебры $\mathscr{A}_{\mu}\left(z_{1}, \ldots, z_{n}\right)$ в пространстве $V_{(\lambda)}$. Мы докажем, что представление алгебры $\mathscr{A}_{\mu}\left(z_{1}, \ldots, z_{n}\right)$ в 
пространстве $V_{(\lambda)}$ получается предельным переходом из представления алгебры $\mathscr{A}\left(z_{1}, \ldots, z_{n+1}\right)$ в пространстве $\left[V_{(\lambda)} \otimes M_{z_{n+1} \mu}^{*}\right]^{\operatorname{sing}}$, состоящем из особых относительно $\operatorname{diag}_{n+1}(\mathfrak{g})$ векторов в $U(\mathfrak{g})^{\otimes(n+1)}$-модуле $V_{(\lambda)} \otimes M_{z_{n+1} \mu}^{*}$ при $z_{n+1} \rightarrow \infty$ (где $M_{z_{n+1} \mu}^{*}$ - модуль, контраградиентный к модулю Верма со старшим весом $\left.z_{n+1} \mu\right)$. Таким образом, представление алгебры $\mathscr{A}_{\mu}\left(z_{1}, \ldots, z_{n}\right)$ в пространстве $V_{(\lambda)}$ есть, в некотором смысле, предельный случай модели Годена.

Мы проверим гипотезу о простоте спектра для представления алгебры $\mathscr{A}_{\mu}\left(z_{1}, \ldots, z_{n}\right)$ в пространстве $V_{(\lambda)}$ в случае $\mathfrak{g}=s l_{r}$. Ключевым в доказательстве является тот факт, что замыкание семейства $\mathscr{A}_{\mu}$ содержит алгебру ГельфандаЦетлина (на уровне пуассоновых алгебр этот факт был доказан Винбергом в [19]). Пользуясь этим, мы докажем, что в случае $\mathfrak{g}=s l_{r}$ для любого представления $V_{(\lambda)}$ при общих значениях параметров $\mu$ и $z_{1}, \ldots, z_{n}$ алгебра $\mathscr{A}_{\mu}\left(z_{1}, \ldots, z_{n}\right)$ имеет простой спектр в $V_{(\lambda)}$. Как следствие мы получим, что спектр алгебры $\mathscr{A}_{0}\left(z_{1}, \ldots, z_{n}\right)$ в пространстве $V_{(\lambda)}^{\text {sing }}$ прост при общих значениях $z_{i}$ и $(\lambda)$.

Работа построена следующим образом. В $\S 2$ и 3 собраны известные факты о подалгебрах Мищенко-Фоменко и центре $Z(\hat{\mathfrak{g}})$ на критическом уровне соответственно. В $\S 4$ описана конструкция подалгебр $\mathscr{A}_{\mu}$ и доказано, что gr $\mathscr{A}_{\mu}=A_{\mu}$. В $\S 5$ описана общая конструкция подалгебр $\mathscr{A}_{\mu}\left(z_{1}, \ldots, z_{n}\right) \subset U(\mathfrak{g})^{\otimes n}$ и доказано, что эти подалгебры имеют максимально возможную степень трансцендентности. В $\S 6$ дано описание представления алгебры $\mathscr{A}_{\mu}\left(z_{1}, \ldots, z_{n}\right)$ в пространстве $V_{(\lambda)}$ как «предельной» модели Годена. Наконец, в $\S 7$ доказаны утверждения о простоте спектра в случае $\mathfrak{g}=s l_{r}$.

Я благодарю Б. Л. Фейгина, Э. Б. Винберга, В. В. Шувалова, А. В. Червова и Д. В. Талалаева за полезные обсуждения.

\section{§2. Метод сдвига инвариантов}

Метод сдвига инвариантов является частным случаем следующей конструкции (см., например, [1]). Пусть $R$ - коммутативная алгебра с двумя согласованными скобками Пуассона $\{\cdot, \cdot\}_{1}$ и $\{\cdot, \cdot\}_{2}$ (т. е. любая линейная комбинация скобок Пуассона $\{\cdot, \cdot\}_{1}$ и $\{\cdot, \cdot\}_{2}$ есть скобка Пуассона). Пусть $Z_{t}$ - центр алгебры $R$ относительно скобки $\{\cdot, \cdot\}_{1}+t\{\cdot, \cdot\}_{2}$. Пусть $A$ - подалгебра в $R$, порожденная всеми $Z_{t}$ для $t$ общего положения.

ФАкт $1[1$, предложение 4]. Подалгебра $A \subset R$ коммутативна относительно каждой скобки $\{\cdot, \cdot\}_{1}+t\{\cdot, \cdot\}_{2}$.

ДоказАтельство. Пусть $a \in Z_{t_{1}}, b \in Z_{t_{2}}$, причем $t_{1} \neq t_{2}$. Тогда линейная по $t$ функция $\{a, b\}_{1}+t\{a, b\}_{2}$ обращается в нуль в двух различных точках $t_{1}$ и $t_{2}$. Следовательно, $\{a, b\}_{1}+t\{a, b\}_{2}=0$ для любого $t$.

Пусть теперь $a, b \in Z_{t_{0}}$. Тогда (в силу того, что $t_{0}$ - значение общего положения) существует непрерывная функция $a(s)$, такая, что $a\left(t_{0}\right)=a$ и $a(s) \in$ $Z_{s}$ в некоторой окрестности значения $t_{0}$. По доказанному имеем $\{a(s), b\}_{1}+$ $t\{a(s), b\}_{2}=0$ для $s$, принадлежащих проколотой окрестности значения $t_{0}$. Следовательно, $\{a, b\}_{1}+t\{a, b\}_{2}=0$.

СлЕДСТВИЕ 1. Пусть $Z S(\mathfrak{g})$ - центр алгебры $S(\mathfrak{g})$ относителъно скобки Пуассона-Ли, и пусть $\mu \in \mathfrak{g}^{*}$. Тогда подалгебра $A_{\mu} \subset S(\mathfrak{g})$, порожденная элементами вида $\partial_{\mu}^{n} \Phi$, где $\Phi \in Z S(\mathfrak{g})$ (или, что то же самое, иентральными 
элементами алгебры $\mathbb{C}\left[\mathfrak{g}^{*}\right]$, сдвинутыми на $t \mu$ при всевозможсных $\left.t \in \mathbb{C}\right)$, коммутативна относительно скобки Пуассона-Ли.

Доказательство. Возьмем в качестве $\{\cdot, \cdot\}_{1}$ скобку Пуассона-Ли, а в качестве $\{\cdot, \cdot\}_{2}$ скобку «с замороженным аргументом», заданную на образующих таK:

$$
\{x, y\}_{2}=\mu([x, y]), \quad x, y \in \mathfrak{g} .
$$

Тогда алгебра $Z_{t}$ порождена центральными элементами алгебры $\mathbb{C}\left[\mathfrak{g}^{*}\right]=S(\mathfrak{g})$, сдвинутыми на $t \mu$.

Так как алгебра Ли $\mathfrak{g}$ полупроста, мы можем отождествить ее с $\mathfrak{g}^{*}$ и считать, что $\mu \in \mathfrak{g}$.

ФАКТ 2 [10]. Если $\mu \in \mathfrak{g}$ - регулярный полупростой элемент, то $A_{\mu}$ есть свободная коммутативная подалгебра в $S(\mathfrak{g})$ с $\frac{1}{2}(\operatorname{dim} \mathfrak{g}+\mathrm{rk} \mathfrak{g})$ образующими ( $и$, таким образом, имеет максимально возможсную степень трансиендентности). В качестве свободных образующих алгебры $A_{\mu}$ могут быть выбраны элементы $\partial_{\mu}^{n} \Phi_{k}, k=1, \ldots, \operatorname{rkg}, n=0,1, \ldots, \operatorname{deg} \Phi_{k}$, где $\Phi_{k}$ - базиснье $\mathfrak{g}$-инварианты в $S(\mathfrak{g})$.

В работе [15] Шуваловым получено описание замыкания семейства подалгебр $A_{\mu} \subset S(\mathfrak{g}), \mu \in \mathfrak{h}^{\text {reg }}$ (т. е. параметр $\mu$ лежит в фиксированной подалгебре Картана). В частности, доказано следующее утверждение.

ФАКТ 3. Пусть $\mu(t)=\mu_{0}+t \mu_{1}+t^{2} \mu_{2}+\cdots \in \mathfrak{h}^{\text {reg }}$ nри $t$ общего положсения. Положим $\mathfrak{z} k=\bigcap_{i=0}^{k} \mathfrak{z} \mathfrak{g}\left(\mu_{i}\right)$ (где $\mathfrak{z} \mathfrak{g}\left(\mu_{i}\right)$ - централизатор в $\mathfrak{g}$ элемента $\left.\mu_{i}\right)$, $\mathfrak{z}-1=\mathfrak{g}$. Тогда

(1) подалгебра $\lim _{t \rightarrow 0} A_{\mu(t)} \subset S(\mathfrak{g})$ порождена элементами $S\left(\mathfrak{z}_{k}\right)^{\mathfrak{z}_{k}}$ u их производными (любого порядка) вдоль $\mu_{k+1}$ для всех $k$;

(2) алгебра $\lim _{t \rightarrow 0} A_{\mu(t)}$ свободна; в качестве свободных образуюших можно взять некоторые производные образующих алгебр $S\left(\mathfrak{z}_{k}\right)^{\mathfrak{z} k}$ вдоль $\mu_{k+1}$.

В частности, это означает, что замыкание семейства $A_{\mu}$ в случае $\mathfrak{g}=s l_{r}$ содержит алгебру Гельфанда-Цетлина (см. [19, п. 6.1-6.4]). Мы разберем этот случай в $§ 7$.

Следующие результаты получены А. А. Тарасовым.

ФАКТ 4 [17]. Подалгебрь $A_{\mu}$ и предельные подалгебрьь вида $\lim _{t \rightarrow 0} A_{\mu(t)}$ являются максимальными коммутативными подалгебрами, т. е. совпадают со своими иентрализаторами в $S(\mathfrak{g})$ относительно скобки Пуассона.

Отображение симметризации $\sigma: S(\mathfrak{g}) \rightarrow U(\mathfrak{g})$ однозначно задается следующим свойством:

$$
\sigma\left(x^{k}\right)=x^{k} \quad \forall x \in \mathfrak{g}, k=0,1,2, \ldots
$$

ФАКТ $5[16,18]$. B случае $\mathfrak{g}=s l_{r}$ образующие подалгебр $A_{\mu}$ и предельных подалгебр вида $\lim _{t \rightarrow 0} A_{\mu(t)}$ могут быть подняты до коммутирующих элементов в $U(\mathfrak{g})$ при помощи отображения симметризации. Это поднятие подалгебр $A_{\mu}$ в универсальную обертььвющую алгебру единственно.

ЗАмЕчАНИЕ 1. В [16] указан явный вид поднимаемых образующих подалгебр $A_{\mu}$ и предельных подалгебр вида $\lim _{t \rightarrow 0} A_{\mu(t)}$. Это, с точностью до пропорциональности, элементы $\partial_{\mu}^{n} \Phi_{k}, k=1, \ldots, r-1, n=0,1, \ldots, \operatorname{deg} \Phi_{k}$ (где $\Phi_{k}-$ элементы из $S\left(s l_{r}\right) s l_{r}$, являющиеся как функции на $s l_{r}$ коэффициентами харак- 
теристического многочлена), и их пределы соответственно. Для нас будет существенно только то, что этот выбор образующих с точностью до пропорциональности непрерывен по параметру $\mu$.

\section{§3. Центр на критическом уровне}

Пусть $\hat{\mathfrak{g}}$ - аффинная алгебра Каца-Муди, соответствующая алгебре Ли $\mathfrak{g}$. Алгебра Ли $\hat{\mathfrak{g}}$ есть центральное расширение алгебры $\mathfrak{g}((t))$ токов на окружности со значениями в $\mathfrak{g}$ с помощью элемента $K$. Коммутационные соотношения заданы следующим образом:

$$
\left[g_{1} \otimes x(t), g_{2} \otimes y(t)\right]=\left[g_{1}, g_{2}\right] \otimes x(t) y(t)+\kappa_{c}\left(g_{1}, g_{2}\right) \operatorname{Res}_{t=0} x(t) d y(t) \cdot K,
$$

где $\kappa_{c}$ - инвариантное скалярное произведение на $\mathfrak{g}$, заданное формулой

$$
\kappa_{c}\left(g_{1}, g_{2}\right)=-\frac{1}{2} \operatorname{Tr}_{\mathfrak{g}} \operatorname{ad}\left(g_{1}\right) \operatorname{ad}\left(g_{2}\right) .
$$

Положим $\hat{\mathfrak{g}}_{+}=\mathfrak{g}[[t]] \subset \hat{\mathfrak{g}}$ и $\hat{\mathfrak{g}}_{-}=t^{-1} \mathfrak{g}\left[t^{-1}\right] \subset \hat{\mathfrak{g}}$.

Определим пополнение $\widetilde{U}(\hat{\mathfrak{g}})$ универсальной обертывающей алгебры $U(\hat{\mathfrak{g}})$ как обратный предел факторпространств $U(\hat{\mathfrak{g}}) / U(\hat{\mathfrak{g}})\left(t^{n} \mathfrak{g}[[t]]\right), n>0$. Действие алгебры $\widetilde{U}(\hat{\mathfrak{g}})$ корректно определено на $\hat{\mathfrak{g}}$-модулях из категории $\mathscr{O}^{0}$ (т. е. на $\hat{\mathfrak{g}}$-модулях, на которых подалгебра $\hat{\mathfrak{g}}_{+}$действует локально конечно). Положим $\widetilde{U}(\hat{\mathfrak{g}})_{c}=$ $\widetilde{U}(\hat{\mathfrak{g}}) /(K-1)$. Эта алгебра действует на $\hat{\mathfrak{g}}$-модулях критического уровня (т. е. $\hat{\mathfrak{g}}$-модулях из категории $\mathscr{O}^{0}$, на которых элемент $K$ действует как единица). Название «критический» обусловлено тем, что теория представлений на этом уровне устроена наиболее сложно. Частным проявлением этого является тот факт, что алгебра $\widetilde{U}(\hat{\mathfrak{g}})_{c}$ имеет нетривиальный центр $Z(\hat{\mathfrak{g}})$. Следующий факт показывает, что этот центр достаточно велик.

Факт 6 [4]. (1) Eстественный гомоморфизм $Z(\hat{\mathfrak{g}}) \rightarrow\left(U(\hat{\mathfrak{g}}) / U(\hat{\mathfrak{g}})\left(\hat{\mathfrak{g}}_{+}+\mathbb{C}(K-\right.\right.$ $1)))^{\hat{\mathfrak{g}}_{+}}$сюргективен.

(2) Фильтрачия Пуанкаре-Биркгофа-Витта на универсальной обертывающей алгебре задает фильтрацию на $\hat{\mathfrak{g}}_{+}$-модуле $U(\hat{\mathfrak{g}}) / U(\hat{\mathfrak{g}})\left(\hat{\mathfrak{g}}_{+}+\mathbb{C}(K-1)\right)$. Относительно этой бильтрачии имеем $\operatorname{gr}\left(U(\hat{\mathfrak{g}}) / U(\hat{\mathfrak{g}})\left(\hat{\mathfrak{g}}_{+}+\mathbb{C}(K-1)\right)\right)^{\hat{\mathfrak{g}}_{+}}=$ $\left(S(\hat{\mathfrak{g}}) / S(\hat{\mathfrak{g}})\left(\hat{\mathfrak{g}}_{+}+\mathbb{C} K\right)\right)^{\hat{\mathfrak{g}}_{+}}$.

Дадим теперь явное описание алгебры $\left(S(\hat{\mathfrak{g}}) / S(\hat{\mathfrak{g}})\left(\hat{\mathfrak{g}}_{+}+\mathbb{C} K\right)\right)^{\hat{\mathfrak{g}}_{+}}$. Так как $\hat{\mathfrak{g}}=\hat{\mathfrak{g}}_{+} \oplus \hat{\mathfrak{g}}_{-} \oplus \mathbb{C} K$ как векторное пространство, то каждый элемент пространства $U(\hat{\mathfrak{g}}) / U(\hat{\mathfrak{g}})\left(\hat{\mathfrak{g}}_{+}+\mathbb{C}(K-1)\right)$ (соответственно $\left.S(\hat{\mathfrak{g}}) / S(\hat{\mathfrak{g}})\left(\hat{\mathfrak{g}}_{+}+\mathbb{C} K\right)\right)$ имеет единственный представитель в $U\left(\hat{\mathfrak{g}}_{-}\right)$(соответственно в $\left.S\left(\hat{\mathfrak{g}}_{-}\right)\right)$. Таким образом, имеем естественные вложения

$$
\left(U(\hat{\mathfrak{g}}) / U(\hat{\mathfrak{g}})\left(\hat{\mathfrak{g}}_{+}+\mathbb{C}(K-1)\right)\right)^{\hat{\mathfrak{g}}_{+}} \hookrightarrow U\left(\hat{\mathfrak{g}}_{-}\right)
$$

и

$$
\left(S(\hat{\mathfrak{g}}) / S(\hat{\mathfrak{g}})\left(\hat{\mathfrak{g}}_{+}+\mathbb{C} K\right)\right)^{\hat{\mathfrak{g}}_{+}} \hookrightarrow S\left(\hat{\mathfrak{g}}_{-}\right) .
$$

Пусть $\mathscr{A} \subset U\left(\hat{\mathfrak{g}}_{-}\right)$и $A \subset S\left(\hat{\mathfrak{g}}_{-}\right)$- образы этих вложений. Рассмотрим следующие дифференцирования алгебры Ли $\hat{\mathfrak{g}}_{-}$:

$$
\begin{aligned}
\partial_{t}\left(g \otimes t^{m}\right) & =m g \otimes t^{m-1} & \forall g \in \mathfrak{g}, m & =-1,-2, \ldots, \\
t \partial_{t}\left(g \otimes t^{m}\right) & =m g \otimes t^{m} & \forall g & \in \mathfrak{g}, m=-1,-2, \ldots .
\end{aligned}
$$


Дифференцирования (6), (7) продолжаются до дифференцирований алгебры $S\left(\hat{\mathfrak{g}}_{-}\right)$, а также алгебры $U\left(\hat{\mathfrak{g}}_{-}\right)$. Дифференцирование $(7)$ задает на этих алгебрах градуировку.

Пусть $i_{-1}: S(\mathfrak{g}) \hookrightarrow S\left(\hat{\mathfrak{g}}_{-}\right)$- вложение, переводящее $g \in \mathfrak{g}$ в $g \otimes t^{-1}$. Пусть $\Phi_{k}, k=1, \ldots, \mathrm{rk} \mathfrak{g}$, - образующие алгебры инвариантов $S(\mathfrak{g})^{\mathfrak{g}}$.

ФАКТ $7[2,6,13]$. Подалгебра $A \subset S\left(\hat{\mathfrak{g}}_{-}\right)$свободно порождена элементами $\partial_{t}^{n} \bar{S}_{k}, k=1, \ldots, \operatorname{rkg} \mathfrak{g}, n=0,1,2, \ldots$, где $\bar{S}_{k}=i_{-1}\left(\Phi_{k}\right)$.

Из факта 6 следует, что образующие $\bar{S}_{k}$ поднимаются до (коммутирующих) образующих алгебры $\mathscr{A}$. Иначе говоря, имеет место

СлЕДСТВиЕ 2. (1) Существуют такие однородные относительно менты $S_{k} \in \mathscr{A}$, что gr $S_{k}=\bar{S}_{k}$.

(2) Алгебра $\mathscr{A}$ есть свободная коммутативная алгебра, порожденная элементами $\partial_{t}^{n} S_{k}, k=1, \ldots, \operatorname{rkg}, n=0,1,2, \ldots$

В дальнейших рассмотрениях мы будем использовать только существование коммутативной подалгебры $\mathscr{A} \subset U\left(\hat{\mathfrak{g}}_{-}\right)$и это ее описание.

ЗАмЕчАниЕ 2. На данный момент не известно общей формулы для элементов $S_{k}$. Для $\Phi_{1}$ (квадратичного элемента Казимира) соответствующий элемент $S_{1} \in \mathscr{A}$ получается симметризацией из $\bar{S}_{1}=i_{-1}\left(\Phi_{1}\right)$.

ЗАмЕчАниЕ 3. Конструкция высших гамильтонианов Годена устроена так: коммутативная подалгебра $\mathscr{A}\left(z_{1}, \ldots, z_{n}\right) \subset U(\mathfrak{g})^{\otimes n}$ получается как образ подалгебры $\mathscr{A} \subset U\left(\hat{\mathfrak{g}}_{-}\right)$при гомоморфизме специализации $U\left(\hat{\mathfrak{g}}_{-}\right) \rightarrow U(\mathfrak{g})^{\otimes n}$ в точках $z_{1}, \ldots, z_{n}$ (см. [7], [3]). Мы остановимся на этом подробнее в $\S 5$.

\section{§4. Максимальные коммутативные подалгебры в $U(\mathfrak{g})$}

Для любого $z \neq 0$ имеется гомоморфизм

$$
\phi_{z}: U\left(\hat{\mathfrak{g}}_{-}\right) \rightarrow U(\mathfrak{g}), \quad g \otimes t^{m} \mapsto z^{m} g .
$$

Кроме того, имеется гомоморфизм

$$
\phi_{\infty}: U\left(\hat{\mathfrak{g}}_{-}\right) \rightarrow S(\mathfrak{g}), \quad g \otimes t^{-1} \mapsto g, \quad g \otimes t^{m} \mapsto 0, \quad m=-2,-3, \ldots
$$

Пусть $\Delta: U\left(\hat{\mathfrak{g}}_{-}\right) \hookrightarrow U\left(\hat{\mathfrak{g}}_{-}\right) \otimes U\left(\hat{\mathfrak{g}}_{-}\right)-$коумножение. Для любого $z \neq 0$ имеется следующий гомоморфизм:

$$
\phi_{z, \infty}=\left(\phi_{z} \otimes \phi_{\infty}\right) \circ \Delta: U\left(\hat{\mathfrak{g}}_{-}\right) \rightarrow U(\mathfrak{g}) \otimes S(\mathfrak{g}) .
$$

Более явно,

$$
\phi_{z, \infty}\left(g \otimes t^{m}\right)=z^{m} g \otimes 1+\delta_{-1, m} \otimes g
$$

Положим

$$
\mathscr{A}(z, \infty)=\phi_{z, \infty}(\mathscr{A}) \subset U(\mathfrak{g}) \otimes S(\mathfrak{g}) .
$$

ПРЕДЛОЖЕНИЕ 1. Подалгебра $\mathscr{A}(z, \infty)$ порождена коэфбиииентами главньх частей рядов Лорана функиий $S_{k}(w)=\phi_{w-z, \infty}\left(S_{k}\right)$ в точке z и значениями этих функиий в точке $\infty$.

ДокАзАтельство. В самом деле, подалгебра $\mathscr{A}(z, \infty)$ порождена элементами $\phi_{z, \infty}\left(\partial_{t}^{n} S_{k}\right)$. Эти элементы есть коэффициенты ряда Тейлора функции $S_{k}(w)=\phi_{w-z, \infty}\left(S_{k}\right)$ в точке $w=0$. Так как функция $S_{k}(w)$ имеет единственный полюс в точке $z$, то коэффициенты ряда Тейлора функции $S_{k}(w)$ в точке 
$w=0$ линейно выражаются через коэффициенты главной части ряда Лорана этой функции в точке $z$ и ее значение в точке $\infty$, и наоборот.

СлЕДСТВИЕ 3. Подалгебра $\mathscr{A}(z, \infty) \subset U(\mathfrak{g}) \otimes S(\mathfrak{g})$ не зависит от $z$.

ДокАзАтЕльство. В самом деле, коэффициенты главных частей рядов Лорана функций $S_{k}(w)=\phi_{w-z, \infty}\left(S_{k}\right)$ в точке $z$ и значения этих функций в точке $\infty$ не зависят от $z$.

Каждый элемент $\mu \in \mathfrak{g}^{*}$ задает гомоморфизм $S(\mathfrak{g}) \rightarrow \mathbb{C}$ «специализации в точке $\mu \gg$, который мы также будем обозначать буквой $\mu$. Рассмотрим следующее семейство коммутативных подалгебр в $U(\mathfrak{g})$, параметризованное с помощью $\mu \in \mathfrak{g}^{*}:$

$$
\mathscr{A}_{\mu}:=(\mathrm{id} \otimes \mu)(\mathscr{A}(z, \infty)) \subset U(\mathfrak{g}) \text {. }
$$

ПрЕДЛОЖЕНИЕ 2. Элементы подалгебры $\mathscr{A}_{\mu} \subset U(\mathfrak{g})$ инвариантны относителъно действия иентрализатора $\mathfrak{z} g(\mu)$ элемента $\mu$ в $\mathfrak{g}$.

Доказательство. В самом деле, $\mathscr{A}(z, \infty) \subset[U(\mathfrak{g}) \otimes S(\mathfrak{g})]^{\Delta(\mathfrak{g})}$, а гомоморфизм $\mu \mathfrak{z}_{\mathfrak{g}}(\mu)$-эквивариантен. Отсюда следует, что $\mathscr{A}_{\mu} \subset U(\mathfrak{g})^{\mathfrak{z} g}(\mu)$.

Мы теперь докажем, что подалгебры $\mathscr{A}_{\mu} \subset U(\mathfrak{g})$ дают квантование подалгебр Мищенко-Фоменко в $S(\mathfrak{g})$, получаемых методом сдвига инвариантов.

Теорема 1. Для регулярных полупростых элементов $\mu \in \mathfrak{g}^{*}$ имеют место равенства gr $\mathscr{A}_{\mu}=A_{\mu}$.

ДокАЗАтЕльство. Рассмотрим $\mathfrak{g}$-инвариантное дифференцирование $E$ алгебры $U\left(\hat{\mathfrak{g}}_{-}\right) \otimes S(\mathfrak{g})$, заданное на образующих следующим образом:

$$
E((g \otimes x(t)) \otimes 1)=1 \otimes g \operatorname{Res}_{t=0} x(t) d t, \quad E(1 \otimes g)=0 \quad \forall g \in \mathfrak{g} .
$$

Иначе говоря,

$$
\left(g \otimes t^{-m}\right) \otimes 1 \mapsto \delta_{-1, m} \otimes g, \quad 1 \otimes g \mapsto 0 \quad \forall g \in \mathfrak{g} .
$$

ЛЕмма 1. Подалгебра $\mathscr{A}(z, \infty) \subset U(\mathfrak{g}) \otimes S(\mathfrak{g})$ порождена элементами

$$
\left(\phi_{z} \otimes \mathrm{id}\right)\left(E^{j}\left(S_{k} \otimes 1\right)\right) \in U(\mathfrak{g}) \otimes S^{j}(\mathfrak{g}) .
$$

ДокАЗАтельство. Заметим, что

$$
\left(\mathrm{id} \otimes \phi_{\infty} \circ \Delta\right)\left(\partial_{t}^{n} S_{k}\right)=(\exp E)\left(\partial_{t}^{n} S_{k}\right) \in U\left(\hat{\mathfrak{g}}_{-}\right) \otimes S(\mathfrak{g}) .
$$

Так как элементы $S_{k}$ однородны относительно $t \partial_{t}$, то указанные элементы $\left(\phi_{z} \otimes\right.$ $\mathrm{id})\left(E^{j}\left(S_{k} \otimes 1\right)\right)$ суть коэффициенты ряда Лорана функций $S_{k}(w)=\phi_{w-z, \infty}\left(S_{k}\right)=$ $\phi_{w-z}\left((\exp E)\left(S_{k} \otimes 1\right)\right)$ в точке $w=z$. Теперь воспользуемся предложением 1.

Рассмотрим $\mathfrak{g}$-инвариантное дифференцирование $e$ алгебры $S(\mathfrak{g}) \otimes S(\mathfrak{g})$, заданное на образующих следующим образом:

$$
e(g \otimes 1)=1 \otimes g, \quad e(1 \otimes g)=0 .
$$

Очевидно, что для любого $f \in S(\mathfrak{g})$ выполнено равенство $(\mathrm{id} \otimes \mu) \circ e^{j}(f \otimes 1)$ $=\partial_{\mu}^{j} f$.

Заметим теперь, что

$$
\operatorname{gr}\left(\phi_{z} \otimes \mathrm{id}\right)\left(E^{j}\left(S_{k} \otimes 1\right)\right)=z^{\left(-\operatorname{deg} \Phi_{k}+j\right)} e^{j}\left(\Phi_{k} \otimes 1\right) \in S(\mathfrak{g}) \otimes S^{j}(\mathfrak{g}),
$$

так как gr $S_{k}=i_{-1}\left(\Phi_{k}\right)$. Отсюда

$$
\operatorname{gr}(\mathrm{id} \otimes \mu) \circ\left(\phi_{z} \otimes \mathrm{id}\right)\left(E^{j}\left(S_{k} \otimes 1\right)\right)=z^{\left(-\operatorname{deg} \Phi_{k}+j\right)} \partial_{\mu}^{j}\left(\Phi_{k}\right) .
$$


Но элементы $\partial_{\mu}^{j}\left(\Phi_{k}\right)$ порождают подалгебру $A_{\mu}$. Значит, gr $\mathscr{A}_{\mu} \supset A_{\mu}$. Так как элементы $\partial_{\mu}^{j}\left(\Phi_{k}\right)$ алгебраически независимы (факт 2$)$, а из леммы следует, что элементы $(\mathrm{id} \otimes \mu) \circ\left(\phi_{z} \otimes \mathrm{id}\right)\left(E^{j}\left(S_{k} \otimes 1\right)\right)$ порождают алгебру $\mathscr{A}_{\mu}$, то gr $\mathscr{A}_{\mu}=A_{\mu}$.

\section{§5. Коммутативные подалгебры в $U(\mathfrak{g})^{\otimes n}$}

Теперь рассмотрим более общую конструкцию. Пусть $U(\mathfrak{g})^{\otimes n}-$ тензорное произведение $n$ экземпляров универсальной обертывающей алгебры $U(\mathfrak{g})$. Мы будем обозначать через $\mathfrak{g}^{(i)}$ подпространство $1 \otimes \cdots \otimes 1 \otimes \mathfrak{g} \otimes 1 \otimes \cdots \otimes 1 \subset U(\mathfrak{g})^{\otimes n}$, где $\mathfrak{g}$ стоит на $i$-м месте. Соответственно для элемента $u \in U(\mathfrak{g})$ мы положим

$$
u^{(i)}=1 \otimes \cdots \otimes 1 \otimes u \otimes 1 \otimes \cdots \otimes 1 \in U(\mathfrak{g})^{\otimes n} .
$$

Пусть $\operatorname{diag}_{n}: U\left(\hat{\mathfrak{g}}_{-}\right) \hookrightarrow U\left(\hat{\mathfrak{g}}_{-}\right)^{\otimes n}-$ диагональное вложение. Для каждого набора попарно различных $z_{i}, i=1, \ldots, n$, имеется следующий гомоморфизм:

$$
\phi_{z_{1}, \ldots, z_{n}, \infty}=\left(\phi_{z_{1}} \otimes \cdots \otimes \phi_{z_{n}} \otimes \phi_{\infty}\right) \circ \operatorname{diag}_{n+1}: U\left(\hat{\mathfrak{g}}_{-}\right) \rightarrow U(\mathfrak{g})^{\otimes n} \otimes S(\mathfrak{g}) .
$$

Более явно,

$$
\phi_{z_{1}, \ldots, z_{n}, \infty}\left(g \otimes t^{m}\right)=\sum_{i=1}^{n} z_{i}^{m} g^{(i)} \otimes 1+\delta_{-1, m} \otimes g .
$$

Положим

$$
\mathscr{A}\left(z_{1}, \ldots, z_{n}, \infty\right)=\phi_{z_{1}, \ldots, z_{n}, \infty}(\mathscr{A}) \subset U(\mathfrak{g})^{\otimes n} \otimes S(\mathfrak{g}) .
$$

Следующее утверждение доказывается аналогично предложению 1 и следствию 2.

ПредлОЖенИЕ 3. (1) Подалгебры $\mathscr{A}\left(z_{1}, \ldots, z_{n}, \infty\right)$ порождены коэфбициентами главных частей рядов Лорана функиий

$$
S_{k}\left(w ; z_{1}, \ldots, z_{n}\right)=\phi_{w-z_{1}, \ldots, w-z_{n}, \infty}\left(S_{k}\right)
$$

в точках $z_{1}, \ldots, z_{n}$ и значениями этих функиий в точке $\infty$.

(2) Подалгебры $\mathscr{A}\left(z_{1}, \ldots, z_{n}, \infty\right)$ не меняются при одновременной афбинной замене параметров $z_{i} \mapsto a z_{i}+b$.

(3) Элементы подалгебр $\mathscr{A}\left(z_{1}, \ldots, z_{n}, \infty\right)$ инвариантны относительно диагонального действия алгебры Ли $\mathfrak{g}$.

Рассмотрим следующее семейство коммутативных подалгебр в $U(\mathfrak{g})^{\otimes n}$, параметризованное числами $z_{1}, \ldots, z_{n} \in \mathbb{C}$ и элементами $\mu \in \mathfrak{g}^{*}:$

$$
\mathscr{A}_{\mu}\left(z_{1}, \ldots, z_{n}\right):=(\mathrm{id} \otimes \mu)\left(\mathscr{A}\left(z_{1}, \ldots, z_{n}, \infty\right)\right) \subset U(\mathfrak{g})^{\otimes n} .
$$

Как непосредственное следствие предложения 3 получаем

ПредлОЖениЕ 4 . (1) Подалгебры $\mathscr{A}_{\mu}\left(z_{1}, \ldots, z_{n}\right)$ не меняются при одновременном сдвиге параметров $z_{i} \mapsto z_{i}+b$.

(2) Элементы подалгебры $\mathscr{A}_{\mu}\left(z_{1}, \ldots, z_{n}\right)$ инвариантны относительно диагонального действия централизатора $\mathfrak{z} \mathfrak{g}(\mu)$.

ЗАмечАниЕ 4 . Подалгебры $\mathscr{A}_{0}\left(z_{1}, \ldots, z_{n}\right) \subset U(\mathfrak{g})^{\otimes n}$ могут быть получены как образы подалгебры $\mathscr{A} \subset U\left(\hat{\mathfrak{g}}_{-}\right)$при гомоморфизме

$$
\phi_{z_{1}, \ldots, z_{n}}=\left(\phi_{z_{1}} \otimes \cdots \otimes \phi_{z_{n}}\right) \circ \operatorname{diag}_{n}: U\left(\hat{\mathfrak{g}}_{-}\right) \rightarrow U(\mathfrak{g})^{\otimes n} .
$$


Это в точности подалгебры $\mathscr{A}\left(z_{1}, \ldots, z_{n}\right) \subset U(\mathfrak{g})^{\otimes n}$ высших гамильтонианов Годена, построенные в [7] (см. также [3]). Квадратичные гамильтонианы Годена (1) получаются как линейные комбинации элементов $\phi_{z_{1}, \ldots, z_{n}}\left(\partial_{t}^{n} \bar{S}_{1}\right), n=0,1,2, \ldots$.

В дальнейшем мы будем писать $\mathscr{A}\left(z_{1}, \ldots, z_{n}\right)$ вместо $\mathscr{A}_{0}\left(z_{1}, \ldots, z_{n}\right)$.

ПрЕДЛОЖЕНИЕ 5. Подалгебры $\mathscr{A}_{\mu}\left(z_{1}, \ldots, z_{n}\right)$ содерэсат следующие «неоднородные гамильтонианы Годена»:

$$
H_{i}=\sum_{k \neq i} \sum_{a=1}^{\operatorname{dim} \mathfrak{g}} \frac{x_{a}^{(i)} x_{a}^{(k)}}{z_{i}-z_{k}}+\sum_{a=1}^{\operatorname{dim} \mathfrak{g}} \mu\left(x_{a}\right) x_{a}^{(i)} .
$$

Доказательство. Так как элемент $S_{1} \in \mathscr{A}$ получается симметризацией из $\bar{S}_{1}=i_{-1}\left(\Phi_{1}\right)$, то элемент $H_{i}$ есть коэффициент при $1 /\left(z-z_{i}\right)$ в разложении функции $S_{1}\left(w ; z_{1}, \ldots, z_{n}\right)=\phi_{w-z_{1}, \ldots, w-z_{n}, \infty}\left(S_{1}\right)$ в точке $w=z_{i}$. Теперь воспользуемся предложением 3.

Алгебра $U(\mathfrak{g})^{\otimes n} \otimes S(\mathfrak{g})^{\otimes m}$ имеет возрастающую фильтрацию конечномерными пространствами, $U(\mathfrak{g})^{\otimes n} \otimes S(\mathfrak{g})^{\otimes m}=\bigcup_{k=0}^{\infty}\left(U(\mathfrak{g})^{\otimes n} \otimes S(\mathfrak{g})^{\otimes m}\right)_{(k)}$ (по степени выражения через образующие). Для однопараметрического семейства подалгебр $B(s) \subset U(\mathfrak{g})^{\otimes n} \otimes S(\mathfrak{g})^{\otimes m}$ определим предел $\lim _{s \rightarrow \infty} B(s)$ как

$$
\bigcup_{k=0}^{\infty} \lim _{s \rightarrow \infty} B(s) \cap\left(U(\mathfrak{g})^{\otimes n} \otimes S(\mathfrak{g})^{\otimes m}\right)_{(k)} .
$$

Ясно, что предел семейства коммутативных подалгебр есть коммутативная подалгебра. Ясно также, что такие предельные переходы коммутируют с гомоморфизмами фильтрованных алгебр (в частности, с проекциями на любой сомножитель и с конечномерными представлениями).

Теорема 2. Для регулярных полупростых $\mu \in \mathfrak{g}^{*}$ имеет место равенство $\lim _{s \rightarrow \infty} \mathscr{A}_{\mu}\left(s z_{1}, \ldots, s z_{n}\right)=\mathscr{A}_{\mu}^{(1)} \otimes \cdots \otimes \mathscr{A}_{\mu}^{(n)} \subset U(\mathfrak{g})^{\otimes n}$.

ДокАЗАТЕльство. Для доказательства нам понадобится

Лемма 2. $\lim _{z \rightarrow \infty} \phi_{z}=\varepsilon$, где $\varepsilon: U\left(\hat{\mathfrak{g}}_{-}\right) \rightarrow \mathbb{C} \cdot 1 \subset U(\mathfrak{g})-$ коединица.

ДокАЗАтЕЛьство. Достаточно проверить это на образующих. Имеем

$$
\lim _{z \rightarrow \infty} \phi_{z}\left(g \otimes t^{m}\right)=\lim _{z \rightarrow \infty} z^{m} g=0 \quad \forall g \in \mathfrak{g}, m=-1,-2, \ldots
$$

Выберем образующие алгебры $\mathscr{A}\left(s z_{1}, \ldots, s z_{n}, \infty\right)$, как в предложении 3. Коэффициенты ряда Лорана функции $S_{k}\left(w ; s z_{1}, \ldots, s z_{n}\right)$ в точке $s z_{i}$ равны коэффициентам ряда Лорана функции $S_{k}\left(w+s z_{i} ; s z_{1}, \ldots, s z_{n}\right)$ в точке 0 . С другой стороны, из леммы 2 следует, что

$$
\begin{aligned}
\lim _{s \rightarrow \infty} S_{k}(w & \left.+s z_{i} ; s z_{1}, \ldots, s z_{n}\right)=\lim _{s \rightarrow \infty} \phi_{w-s\left(z_{1}-z_{i}\right), \ldots, w, \ldots, w-s\left(z_{n}-z_{i}\right), \infty}\left(S_{k}\right) \\
& =\left(\varepsilon \otimes \cdots \otimes \varepsilon \otimes \phi_{w} \otimes \varepsilon \otimes \cdots \otimes \varepsilon \otimes \phi_{\infty}\right) \circ \operatorname{diag}_{n+1}\left(S_{k}\right)=S_{k}^{(i)}(w ; 0) .
\end{aligned}
$$

Таким образом, образующие алгебры $\mathscr{A}\left(s z_{1}, \ldots, s z_{n}, \infty\right)$ дают в пределе образующие алгебры $\mathscr{A}\left(z_{1}, \infty\right)^{(1)} \cdots \mathscr{A}\left(z_{n}, \infty\right)^{(n)}$. Отсюда следует, что

$$
\lim _{s \rightarrow \infty} \mathscr{A}\left(s z_{1}, \ldots, s z_{n}, \infty\right) \supset \mathscr{A}\left(z_{1}, \infty\right)^{(1)} \cdots \mathscr{A}\left(z_{n}, \infty\right)^{(n)},
$$


а значит, и

$$
\lim _{s \rightarrow \infty} \mathscr{A}_{\mu}\left(s z_{1}, \ldots, s z_{n}\right) \supset \mathscr{A}_{\mu}^{(1)} \otimes \cdots \otimes \mathscr{A}_{\mu}^{(n)} .
$$

Но из факта 4 следует, что подалгебра $\mathscr{A}_{\mu}^{(1)} \otimes \cdots \otimes \mathscr{A}_{\mu}^{(n)} \subset U(\mathfrak{g})^{\otimes n}$ совпадает со своим централизатором. Значит, $\lim _{s \rightarrow \infty} \mathscr{A}_{\mu}\left(s z_{1}, \ldots, s z_{n}\right)=\mathscr{A}_{\mu}^{(1)} \otimes \cdots \otimes \mathscr{A}_{\mu}^{(n)}$.

СлЕДСТВИЕ 4. Коммутативные подалгебры $\mathscr{A}_{\mu}\left(z_{1}, \ldots, z_{n}\right) \subset U(\mathfrak{g})^{\otimes n}$ при общих значениях параметров имеют максимально возможную степень трансцендентности (равную $\left.\frac{n}{2}(\operatorname{dim} \mathfrak{g}+\mathrm{rk} \mathfrak{g})\right)$.

ДокАЗАтЕльство. В самом деле, при общих $\mu$ подалгебра $\mathscr{A}_{\mu}^{(1)} \otimes \cdots \otimes \mathscr{A}_{\mu}^{(n)} \subset$ $U(\mathfrak{g})^{\otimes n}$ имеет максимально возможную степень трансцендентности (это следует из факта 2). Так как такие подалгебры лежат в замыкании семейства $\mathscr{A}_{\mu}\left(z_{1}, \ldots, z_{n}\right)$, то при общих значениях параметров подалгебры $\mathscr{A}_{\mu}\left(z_{1}, \ldots, z_{n}\right)$ также имеют максимально возможную степень трансцендентности.

Рассмотрим однопараметрическое семейство алгебр $U(\mathfrak{g})_{t}$ с пространством образующих $\mathfrak{g}$ и определяющими соотношениями

$$
x y-y x=t[x, y] \quad \forall x, y \in \mathfrak{g} .
$$

При $t \neq 0$ отображение $\mathfrak{g} \rightarrow \mathfrak{g}, x \mapsto t^{-1} x$, индуцирует изоморфизм алгебр

$$
\psi_{t}: U(\mathfrak{g}) \stackrel{\sim}{\longrightarrow} U(\mathfrak{g})_{t} .
$$

При $t=0$ имеем $U(\mathfrak{g})_{0}=S(\mathfrak{g})$.

Рассмотрим коммутативную подалгебру

$$
\left(\mathrm{id}^{\otimes n} \otimes \psi_{z^{-1}}\right)\left(\mathscr{A}\left(z_{1}, \ldots, z_{n}, z\right)\right) \subset U(\mathfrak{g})^{\otimes n} \otimes U(\mathfrak{g})_{z^{-1}} .
$$

В пределе при $z \rightarrow \infty$ получается некоторая коммутативная подалгебра в $U(\mathfrak{g})^{\otimes n} \otimes S(\mathfrak{g})$.

ТеОрема 3. Имеет место равенство

$$
\lim _{z \rightarrow \infty}\left(\mathrm{id}^{\otimes n} \otimes \psi_{z^{-1}}\right)\left(\mathscr{A}\left(z_{1}, \ldots, z_{n}, z\right)\right)=\mathscr{A}\left(z_{1}, \ldots, z_{n}, \infty\right) \subset U(\mathfrak{g})^{\otimes n} \otimes S(\mathfrak{g}) .
$$

ДокАЗАТЕЛьСТво. Предварительно докажем такую лемму:

ЛЕмма 3. $\lim _{z \rightarrow \infty} \psi_{z^{-1}} \circ \phi_{z}=\phi_{\infty}$.

ДоказАтельство. Достаточно проверить это на образующих. Имеем

$$
\psi_{z^{-1}} \circ \phi_{z}\left(g \otimes t^{m}\right)=z \cdot z^{m} g \in U(\mathfrak{g})_{z^{-1}} \quad \forall g \in \mathfrak{g}, m=-1,-2, \ldots
$$

Отсюда

$$
\lim _{z \rightarrow \infty} \psi_{z^{-1}} \circ \phi_{z}\left(g \otimes t^{m}\right)=\delta_{-1, m} g=\psi_{\infty}\left(g \otimes t^{m}\right) \in S(\mathfrak{g}) .
$$

Из леммы 3 следует, что

$$
\begin{aligned}
\lim _{z \rightarrow \infty} & \left(\mathrm{id}^{\otimes n} \otimes \psi_{z^{-1}}\right)\left(\mathscr{A}\left(z_{1}, \ldots, z_{n}, z\right)\right) \\
& =\lim _{z \rightarrow \infty}\left(\phi_{z_{1}} \otimes \cdots \otimes \phi_{z_{n}} \otimes\left(\psi_{z^{-1}} \circ \phi_{z}\right)\right) \circ \operatorname{diag}_{n+1}(\mathscr{A}) \\
& =\lim _{z \rightarrow \infty}\left(\phi_{z_{1}} \otimes \cdots \otimes \phi_{z_{n}} \otimes \phi_{\infty}\right) \circ \operatorname{diag}_{n+1}(\mathscr{A}) \\
& =\mathscr{A}\left(z_{1}, \ldots, z_{n}, \infty\right) \subset U(\mathfrak{g})^{\otimes n} \otimes S(\mathfrak{g}) .
\end{aligned}
$$




\section{§6. «Предельная» модель Годена}

Пусть $V_{\lambda}$ - конечномерный неприводимый $\mathfrak{g}$-модуль со старшим весом $\lambda$. Мы будем рассматривать следующий $U(\mathfrak{g})^{\otimes n}$-модуль:

$$
V_{(\lambda)}:=V_{\lambda_{1}} \otimes \cdots \otimes V_{\lambda_{n}} .
$$

Подалгебра $\mathscr{A}\left(z_{1}, \ldots, z_{n}\right) \subset U(\mathfrak{g})^{\otimes n}$ состоит из $\operatorname{diag}_{n}(\mathfrak{g})$-инвариантных элементов и поэтому действует в подпространстве $V_{(\lambda)}^{\text {sing }} \subset V_{(\lambda)}$ особых векторов относительно $\operatorname{diag}_{n}(\mathfrak{g})$. Это представление алгебры $\mathscr{A}\left(z_{1}, \ldots, z_{n}\right)$ называют (n-точечной) моделью Годена.

Мы покажем, что для полупростого $\mu \in \mathfrak{g}^{*}$ представление подалгебры $\mathscr{A}_{\mu}\left(z_{1}, \ldots, z_{n}\right) \subset U(\mathfrak{g})^{\otimes n}$ в пространстве $V_{(\lambda)}$ есть предельный случай $(n+1)$-точечной модели Годена.

Пусть $M_{\chi}^{*}$ - модуль, контраградиентный к модулю Верма со старшим весом $\chi$. Этот модуль может быть реализован следующим образом. Пусть $\Delta_{+}-$ множество положительных корней алгебры Ли $\mathfrak{g}$. Тогда $M_{\chi}^{*}=\mathbb{C}\left[x_{\alpha}\right]_{\alpha \in \Delta_{+}}$(образующие $x_{\alpha}$ имеют (мульти)степень $\alpha$ ), а элементы алгебры Ли $\mathfrak{g}$ действуют по следующим формулам.

(1) Элементы $e_{\alpha}, \alpha \in \Delta_{+}$, подалгебры $\mathfrak{n}_{+}$действуют как

$$
\frac{\partial}{\partial x_{\alpha}}+\sum_{\beta>\alpha} P_{\beta}^{\alpha} \frac{\partial}{\partial x_{\beta}},
$$

где $P_{\beta}^{\alpha}$ - некоторые многочлены степеней $\beta-\alpha$.

(2) Элементы $h \in \mathfrak{h}$ действуют как

$$
\chi(h)-\sum_{\beta \in \Delta_{+}} \beta(h) x_{\beta} \frac{\partial}{\partial x_{\beta}} .
$$

(3) Образующие $e_{-\alpha_{i}}\left(\alpha_{i}-\right.$ простые корни) подалгебры $\mathfrak{n}_{-}$действуют как

$$
\chi\left(h_{\alpha_{i}}\right) x_{\alpha_{i}}+\sum_{\beta \in \Delta_{+}} Q_{\beta}^{\alpha_{i}} \frac{\partial}{\partial x_{\beta}},
$$

где $Q_{\beta}^{\alpha_{i}}$ - некоторые многочлены степеней $\beta+\alpha_{i}$.

Рассмотрим $U(\mathfrak{g})^{\otimes n} \otimes U(\mathfrak{g})$-модуль $V_{(\lambda)} \otimes M_{z \mu}^{*}$. Отождествим векторное пространство $M_{z \mu}^{*}$ с $\mathbb{C}\left[x_{\alpha}\right]$ и перенормируем образующие, полагая $y_{\alpha}=z^{h t(\alpha)} x_{\alpha}$, где $h t(\alpha)$ - высота корня $\alpha$. Формулы для действия элементов алгебры Ли $\mathfrak{g}$ в $M_{z \mu}^{*}=\mathbb{C}\left[y_{\alpha}\right]$ переписываются следующим образом:

$$
\begin{aligned}
e_{\alpha} & =z^{h t(\alpha)} \frac{\partial}{\partial y_{\alpha}}+\sum_{\beta>\alpha} z^{h t(\alpha)} P_{\beta}^{\alpha} \frac{\partial}{\partial y_{\beta}}, \\
h & =z \mu(h)-\sum_{\beta \in \Delta_{+}} \beta(h) y_{\beta} \frac{\partial}{\partial y_{\beta}}, \\
e_{-\alpha_{i}} & =\mu\left(h_{\alpha_{i}}\right) y_{\alpha_{i}}+z^{-1} \sum_{\beta \in \Delta_{+}} Q_{\beta}^{\alpha_{i}} \frac{\partial}{\partial y_{\beta}} .
\end{aligned}
$$


Таким образом, можно считать базис в пространстве $V_{(\lambda)} \otimes M_{z \mu}^{*}=V_{(\lambda)} \otimes \mathbb{C}\left[y_{\alpha}\right]$ не зависящим от $z$, а операторы из $U(\mathfrak{g})^{\otimes n} \otimes U(\mathfrak{g})$ зависящими от $z$. Подпространство особых векторов $\left[V_{(\lambda)} \otimes M_{z \mu}^{*}\right]^{\operatorname{sing}} \subset V_{(\lambda)} \otimes \mathbb{C}\left[y_{\alpha}\right]$ становится также зависящим от $z$. Далее, пространство $V_{(\lambda)} \otimes M_{z \mu}^{*}=V_{(\lambda)} \otimes \mathbb{C}\left[y_{\alpha}\right]$ градуировано весами диагонального действия алгебры Ли $\mathfrak{g}$, причем однородные компоненты не зависят от $z$ и имеют конечные размерности. Подпространство $\left[V_{(\lambda)} \otimes M_{z \mu}^{*}\right]^{\operatorname{sing}} \subset V_{(\lambda)} \otimes \mathbb{C}\left[y_{\alpha}\right]$ лежит в конечной сумме однородных компонент; поэтому корректно определен предел $\lim _{z \rightarrow \infty}\left[V_{(\lambda)} \otimes M_{z \mu}^{*}\right]^{\operatorname{sing}} \subset V_{(\lambda)} \otimes \mathbb{C}\left[y_{\alpha}\right]$. При этом образ алгебры $\mathscr{A}\left(z_{1}, \ldots, z_{n}, z\right)$ в $\operatorname{End}\left(\left[V_{(\lambda)} \otimes M_{z \mu}^{*}\right]^{\operatorname{sing}}\right)$ в пределе при $z \rightarrow \infty$ дает некоторую коммутативную подалгебру в $\operatorname{End}\left(\lim _{z \rightarrow \infty}\left[V_{(\lambda)} \otimes M_{z \mu}^{*}\right]^{\operatorname{sing}}\right)$.

TEOpEMA 4. При $z \rightarrow \infty$

(1) предел подпространств $\left[V_{(\lambda)} \otimes M_{z \mu}^{*}\right]^{\operatorname{sing}} \subset V_{(\lambda)} \otimes \mathbb{C}\left[y_{\alpha}\right]$ ecms $V_{(\lambda)} \otimes 1$;

(2) предел образа алгебры $\mathscr{A}\left(z_{1}, \ldots, z_{n}, z\right)$ в $\operatorname{End}\left(\left[V_{(\lambda)} \otimes M_{z \mu}^{*}\right]^{\text {sing }}\right)$ содержнит образ алгебры $\mathscr{A}_{\mu}\left(z_{1}, \ldots, z_{n}\right)$ в $\operatorname{End}\left(V_{(\lambda)} \otimes 1\right)=\operatorname{End}\left(V_{(\lambda)}\right)$.

Доказательство. Докажем первое утверждение. Подпространство $\left[V_{(\lambda)} \otimes\right.$ $\left.M_{z \mu}^{*}\right]^{\operatorname{sing}} \subset V_{(\lambda)} \otimes M_{z \mu}^{*}$ есть пересечение ядер операторов $\operatorname{diag}_{n+1}\left(e_{\alpha}\right)=\sum_{i=1}^{n+1} e_{\alpha}^{(i)}$, $\alpha \in \Delta_{+}$. Легко видеть, что

$$
\lim _{z \rightarrow \infty} z^{-h t(\alpha)} \operatorname{diag}_{n+1}\left(e_{\alpha}\right)=1^{\otimes n} \otimes\left(\frac{\partial}{\partial y_{\alpha}}+\sum_{\beta>\alpha} P_{\beta}^{\alpha} \frac{\partial}{\partial y_{\beta}}\right) .
$$

Это означает, что

$$
\lim _{z \rightarrow \infty}\left[V_{(\lambda)} \otimes M_{z \mu}^{*}\right]^{\text {sing }} \subset \bigcap_{\alpha \in \Delta_{+}} \operatorname{Ker} 1^{\otimes n} \otimes\left(\frac{\partial}{\partial y_{\alpha}}+\sum_{\beta>\alpha} P_{\beta}^{\alpha} \frac{\partial}{\partial y_{\beta}}\right)=V_{(\lambda)} \otimes 1 .
$$

Но $\operatorname{dim}\left[V_{(\lambda)} \otimes M_{z \mu}^{*}\right]^{\operatorname{sing}} \geqslant \operatorname{dim} V_{(\lambda)}$. Значит, $\lim _{z \rightarrow \infty}\left[V_{(\lambda)} \otimes M_{z \mu}^{*}\right]^{\operatorname{sing}}=V_{(\lambda)} \otimes 1$.

Докажем второе утверждение. Модуль $V_{(\lambda)} \otimes M_{z \mu}^{*}=V_{(\lambda)} \otimes \mathbb{C}\left[y_{\alpha}\right]$ можно

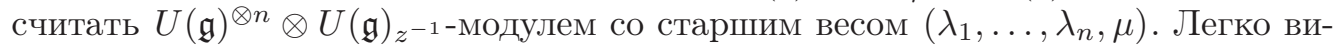
деть (из формул для действия алгебры Ли $\mathfrak{g}$ в $\left.\mathbb{C}\left[y_{\alpha}\right]\right)$, что для простых корней $\alpha_{i} \in \Delta_{+}$

$$
\lim _{z \rightarrow \infty} 1 \otimes \cdots \otimes 1 \otimes e_{-\alpha_{i}}=\lim _{z \rightarrow \infty} z^{-1} 1 \otimes \cdots \otimes 1 \otimes \psi_{z^{-1}}\left(e_{-\alpha_{i}}\right)=0 .
$$

Это значит, что подпространство $V_{(\lambda)} \otimes 1 \subset V_{(\lambda)} \otimes \mathbb{C}\left[y_{\alpha}\right]$ является собственным относительно действия $\lim _{z \rightarrow \infty} U(\mathfrak{g})^{\otimes n} \otimes U(\mathfrak{g})_{z^{-1}}=U(\mathfrak{g})^{\otimes n} \otimes S(\mathfrak{g})$, причем $1 \otimes$ $\cdots \otimes 1 \otimes S(\mathfrak{g})$ действует на нем характером $\mu$. По теореме 3 имеем

$$
\lim _{z \rightarrow \infty}\left(\mathrm{id}^{\otimes n} \otimes \psi_{z^{-1}}\right)\left(\mathscr{A}\left(z_{1}, \ldots, z_{n}, z\right)\right)=\mathscr{A}\left(z_{1}, \ldots, z_{n}, \infty\right) \subset U(\mathfrak{g})^{\otimes n} \otimes S(\mathfrak{g}) .
$$

Это значит, что предел образа алгебры $\mathscr{A}\left(z_{1}, \ldots, z_{n}, z\right)$ в $\operatorname{End}\left(\left[V_{(\lambda)} \otimes M_{z \mu}^{*}\right]^{\operatorname{sing}}\right)$ coдержит образ алгебры $(\mathrm{id} \otimes \mu)\left(\mathscr{A}\left(z_{1}, \ldots, z_{n}, \infty\right)\right)=\mathscr{A}_{\mu}\left(z_{1}, \ldots, z_{n}\right)$ в $\operatorname{End}\left(V_{(\lambda)} \otimes 1\right)$.

\section{$\S 7$. Случай $s l_{r}$}

В этом разделе $\mathfrak{g}=s l_{r}$.

Лемма 4. $B$ случае $\mathfrak{g}=s l_{r} u \mu(t)=E_{11}+t E_{22}+\cdots+t^{n-1} E_{n n} \quad$ nредел $\lim _{t \rightarrow 0} \mathscr{A}_{\mu(t)}$ есть подалгебра Гельфанда-Цетлина в $U\left(s_{r}\right)$. 
Доказательство. Из результатов В. В. Шувалова (факт 3) следует, что ассоциированная градуированная алгебра $\lim _{t \rightarrow 0} A_{\mu(t)} \subset S(\mathfrak{g})$ есть подалгебра Гельфанда-Цетлина в $S(\mathfrak{g})$. В самом деле, $\mathfrak{z} k$ в этом случае есть алгебра Ли $s l_{r-k-1} \oplus \mathbb{C}^{k+1}$, состоящая из матриц $A \in s l_{r}$, таких, что

$$
A_{i j}=A_{j i}=0, \quad i=1, \ldots, k+1, j=1, \ldots, r, i \neq j .
$$

Подалгебра в $S\left(s l_{r}\right)$, порожденная элементами $S(\mathfrak{z} k)^{\mathfrak{z} k}$ при всех $k$, есть подалгебра Гельфанда-Цетлина.

Так как для любого $\mu$ образующие подалгебры $\mathscr{A} \mu$ получаются из образующих подалгебры $A_{\mu}$ симметризацией (факт 5), то и образующие подалгебры $\lim _{t \rightarrow 0} \mathscr{A}_{\mu(t)} \subset U(\mathfrak{g})$ получаются из образующих подалгебры $\lim _{t \rightarrow 0} A_{\mu(t)} \subset S(\mathfrak{g})$ симметризацией.

Из единственности поднятия (факт 5) следует, что $\lim _{t \rightarrow 0} \mathscr{A}_{\mu(t)}$ есть подалгебра в $U\left(s l_{r}\right)$, порожденная элементами $Z U(\mathfrak{z} k)$ при всех $k$, т. е. подалгебра Гельфанда-Цетлина в $U\left(s l_{r}\right)$.

Теорема 5. При общих значениях параметров $\mu$ u $z_{1}, \ldots, z_{n}$ алгебра $\mathscr{A}_{\mu}\left(z_{1}, \ldots, z_{n}\right)$ имеет простой спектр в $V_{(\lambda)}$ для любого набора $(\lambda)$ доминантных иелочисленных весов.

ДоказАтеЛьство. (1) Подалгебра Гельфанда-Цетлина в $U\left(s l_{r}\right)$ имеет простой спектр в $V_{\lambda}$ для любого веса $\lambda$. Это известный классический результат.

(2) Так как подалгебра Гельфанда-Цетлина получается предельным переходом из подалгебр $\mathscr{A}_{\mu}$, то при общих $\mu$ алгебра $\mathscr{A}_{\mu}$ также имеет простой спектр в $V_{\lambda}$ для любого веса $\lambda$.

(3) Отсюда следует, что при общих значениях $\mu$ подалгебра $\mathscr{A}_{\mu}\left(z_{1}\right)^{(1)} \otimes \cdots$ $\otimes \mathscr{A}_{\mu}\left(z_{n}\right)^{(n)}$ имеет простой спектр в $V_{(\lambda)}$. Так как подалгебра $\mathscr{A}_{\mu}\left(z_{1}\right)^{(1)} \otimes \cdots$ $\otimes \mathscr{A}_{\mu}\left(z_{n}\right)^{(n)}$ лежит в замыкании семейства подалгебр $\mathscr{A}_{\mu}\left(z_{1}, \ldots, z_{n}\right)$, то при общих $\mu$ и $z_{i}$ алгебра $\mathscr{A}_{\mu}\left(z_{1}, \ldots, z_{n}\right)$ также имеет простой спектр в $V_{(\lambda)}$.

СледСТвиЕ 5. Существует такое плотное по Зарисскому в $\mathfrak{h}^{*}$ подмножество $W \subset \Lambda_{+} \times \cdots \times \Lambda_{+}\left(\right.$әде $\Lambda_{+}-$множество иелочисленных доминантных весов $)$, что для любого $(\lambda)=\left(\lambda_{1}, \ldots \lambda_{n}\right) \in W$ алгебра Годена $\mathscr{A}\left(z_{1}, \ldots, z_{n}\right)$ имеет простой спектр в $V_{(\lambda)}^{\text {sing }}$ при общих значениях параметров $z_{1}, \ldots, z_{n}$.

ДокАЗАТЕЛЬСтво. При фиксированных $\lambda_{1}, \ldots, \lambda_{n-1}$ условие непростоты спектра алгебры $\mathscr{A}\left(z_{1}, \ldots, z_{n}\right)$ в конечномерном пространстве $\left[V_{\lambda_{1}} \otimes \cdots \otimes V_{\lambda_{n-1}} \otimes\right.$ $\left.M_{\lambda_{n}}^{*}\right]^{\text {sing }}$ при любых значениях параметров $z_{1}, \ldots, z_{n}$ есть алгебраическое условие на $\lambda_{n} \in \mathfrak{h}^{*}$. Из теорем 4 и 5 следует, что это условие не всегда выполнено. Следовательно, для любого набора $\lambda_{1}, \ldots \lambda_{n-1}$ множество таких $\lambda_{n} \in \Lambda_{+}$, что спектр алгебры $\mathscr{A}\left(z_{1}, \ldots, z_{n}\right)$ в $\left[V_{\lambda_{1}} \otimes \cdots \otimes V_{\lambda_{n-1}} \otimes M_{\lambda_{n}}^{*}\right]^{\text {sing }}$ прост при общих значениях параметров $z_{1}, \ldots, z_{n}$, плотно по Зарисскому в $\mathfrak{h}^{*}$. Так как $V_{\lambda_{n}} \subset M_{\lambda_{n}}^{*}$, то для всех таких наборов $\lambda_{1}, \ldots, \lambda_{n}$ спектр алгебры $\mathscr{A}\left(z_{1}, \ldots, z_{n}\right)$ в пространстве $V_{(\lambda)}^{\text {sing }}$ также прост.

\section{ЛитератУРА}

[1] А. В. Болсинов, А. В. Борисов, "Согласованные скобки Пуассона на алгебрах Ли", Матем. заметки, 72:1 (2002), 11-34.

[2] A. Beilinson, V. Drinfeld, Quantization of Hitchin's integrable system and Hecke eigensheaves, Preprint, http://www.ma.utexas.edu/ $\sim$ benzvi/BD. 
[3] B. Enriquez, V. Rubtsov, "Hitchin systems, higher Gaudin Hamiltonians and r-matrices", Math. Res. Lett., 3:3 (1996), 343-357; http://arxiv.org/abs/alg-geom/ 9503010.

[4] B. Feigin, E. Frenkel, "Affine Kac-Moody algebras at the critical level and GelfandDikii algebras", Int. Jour. Mod. Phys., A7, Supplement 1A (1992), 197-215.

[5] E. Frenkel, "Affine Algebras, Langlands Duality and Bethe Ansatz", in: XIth International Congress of Mathematical Physics (Paris, 1994), Internat. Press, Cambridge, MA, 1995, 606-642; http://arxiv.org/q-alg/9506003.

[6] E. Frenkel, "Lectures on Wakimoto modules, opers and the center at the critical level", http://arxiv.org/math.QA/0210029.

[7] B. Feigin, E. Frenkel, N. Reshetikhin, "Gaudin model, Bethe Ansatz and critical level", Comm. Math. Phys., 166 (1994), 27-62; http://arxiv.org/hep-th/9402022.

[8] M. Gaudin, "Diagonalisation d'une classe d'hamiltoniens de spin", J. Physique, 37:10 (1976), 1087-1098.

[9] М. Годен, Волновая функиия Бете, Мир, М., 1987.

[10] А. С. Мищенко, А. Т. Фоменко, "Интегрируемость уравнений Эйлера на полупростых алгебрах Ли", Труды семин. вект. тенз. анал., 19 (1979), 3-94.

[11] A. I. Molev, "Yangians and their applications", in: Handbook of algebra, vol. 3, NorthHolland, Amsterdam, 2003, 907-959; http://arxiv.org/math.QA/0211288.

[12] E. Mukhin, A. Varchenko, Norm of a Bethe vector and the Hessian of the master function, Preprint, http://arxiv.org/math.QA/0402349.

[13] M. Mustata, "Jet schemes of locally complete intersection canonical singularities (with an appendix by D. Eisenbud and E. Frenkel)", Invent. Math., 145 (2001), 397-424; http://arxiv.org/math.AG/0008002.

[14] M. Nazarov, G. Olshanski, "Bethe Subalgebras in Twisted Yangians", Comm. Math. Phys., 178:2 (1996), 483-506; http://arxiv.org/q-alg/9507003.

[15] В. Шувалов, "О пределах подалгебр Мищенко-Фоменко в алгебрах Пуассона полупростых алгебр Ли", Функи. анализ и его прил., 36:4 (2002), 55-64.

[16] А. А. Тарасов, "О некоторых максимальных коммутативных подалгебрах в универсальной обертывающей алгебре алгебры Ли $g l_{n} "$, Матем. сб., 191:9 (2000), $115-122$.

[17] А. А. Тарасов, “Максимальность некоторых коммутативных подалгебр в алгебрах Пуассона полупростых алгебр Ли", УМН, 57:5 (2002), 165-166.

[18] А. А. Тарасов, “О единственности поднятия максимальных коммутативных подалгебр из алгебры Пуассона-Ли в обертывающую алгебру”, Матем. сб., 194:7 (2003), 155-160.

[19] Э. Б. Винберг, “О некоторых коммутативных подалгебрах универсальной обертывающей алгебры”, Изв. АН СССР, сер. матем., 54:1 (1990), 3-25.

Лаборатория Понселе Независимого московского университета и CNRS Московский государственный университет им. М. В. Ломоносова, механико-математический факультет e-mail: leo.rybnikov@gmail.com
Поступило в редакцию 9 апреля 2005 г. 